\title{
Analysis of Continuous Feedback Markov Fluid Queues and Its Applications to Modeling Optical Burst Switching
}

\author{
Mehmet Akif Yazıcı and Nail Akar \\ Dept. of Electrical and Electronics Engineering, Bilkent University \\ 06800, Ankara, Turkey \\ \{yazici,akar\}@ee.bilkent.edu.tr
}

\begin{abstract}
Optical Burst Switching (OBS) has been proposed as a candidate technology for the next-generation Internet. In OBS, packets are assembled into a burst, and a burst control packet is sent in advance to inform and reserve resources at the optical nodes in the path of the burst. In this study, we analyze the horizon-based reservation scheme in OBS using Markov fluid queues. First, we provide a solution to continuous feedback Markov fluid queues, then we model the horizon-based reservation scheme as a continuous feedback Markov fluid queue and numerically study it. We provide numerical examples to validate our model and its solution technique as well as to obtain some insight on the horizon-based reservation mechanism.
\end{abstract}

\section{INTRODUCTION}

Optical Burst Switching (OBS) [1] has been the focus of research in the field of optical networks since it offers a compromise between optical packet switching that has yet to be implemented due to lack of optical buffers, and the relatively IP-unfriendly optical circuit switching. Recognizing the inherent burstiness of broadband multimedia traffic, in the OBS paradigm, a number of packets are merged into a single payload, called a burst. A control packet, called the Burst Control Packet (BCP), is sent in the electronic domain in advance of the burst with relevant information in order to reserve resources for the burst. Optical nodes receiving the $\mathrm{BCP}$ configure themselves to accommodate the burst to arrive, if possible. The burst is then sent in the optical domain after an amount of time, called the offset time.

There are different reservation mechanisms for node configuration. The simplest is the Just-in-Time (JIT) [2] mechanism that reserves a node for an incoming burst and rejects all arrivals until the burst is entirely transmitted. With this immediate reservation method, bursts that would arrive after the node becomes available may be blocked if their BCPs arrive when the node is busy. On the other hand, delayed reservation methods such as Just-Enough-Time (JET) [3], [1] and Horizon [4] keep track of the time that the node will become idle, allowing bursts that will arrive later than this value (scheduling horizon) to be accommodated. Horizon is easier to implement than JET that supports void-filling which is the process of allocating the idle times between consecutive reservations to bursts that can fit in.

The offset time can also be used as a means of providing loss probability-based service differentiation. By assigning larger offset times to high priority clients, their burst blocking probability can be reduced. Yoo and Qiao [5] propose such a scheme and provide a method for finding the required offset time for a certain level of isolation between different priority classes.

Other features of OBS networks are fiber delay lines (FDL) that serve as a sort of buffer, and wavelength converters. Using FDLs, contention can be resolved among multiple bursts that arrive at a node within each other's duration. One of the contending bursts is chosen to be transmitted right away, and the rest is "stored" within FDLs and they are transmitted after the node becomes available, if possible. Wavelength converters are used in multi-wavelength links to direct an incoming burst to a different wavelength channel from which it is arriving on, since the node it arrives is already transmitting another burst on that channel.

The burst blocking probability in OBS networks are studied extensively in different settings. Ref. [6] analyzes JET with generally distributed burst lengths and deterministic offset times under low blocking assumption. Morató et al. [7] investigate the blocking time distribution in the existence of FDLs with multiple wavelength channels. In [8], an M/G/k/k approximation is used to analyze a JET system with exponential burst sizes and complete isolation between the multiple classes. Ref. [9] gives a comparison of JIT, JET and horizon-based reservations based on the Erlang-B loss formula. A similar analysis is carried out in [10]. A slotted OBS-JET approximate model is solved using a non-homogeneous Markov chain in [11]. A JET system with uniformly distributed offset times and deterministic burst lengths is analyzed in [12]. All of these studies assume Poisson burst arrivals. In [13], the horizon reservation scheme is studied on a single-channel system with Poisson arrivals, PH-type distributed burst lengths and deterministic offset times. The analysis is based on Markov fluid queues similar to our study. Moreover, as pointed out in [13], analyses based on the Erlang-B loss formula such as [8], [9], [10] cannot reflect the effects of higher order statistics of the burst lengths, or the offset times.

In this study, we investigate the horizon reservation scheme on a single-channel OBS system. We base our solution on the theory of Markov fluid queues, and study the most general case that the fluid queue framework allows us. In this setting, 
the burst arrivals occur according to a Markovian Arrival Process (MAP), the burst lengths possess a phase-type (PHtype) distribution and the offset times are generally distributed.

It is well known that Internet traffic is bursty and correlated in nature. It is therefore appropriate to use arrivals models that allow autocorrelation, which led us to the choice of MAP as opposed to Poisson process. Offset times are generally accepted to be deterministic. However, bursts who have traversed more hops tend to have smaller offset times. Therefore, it is common that different bursts will have different offset times. Moreover, although the original offset time may be deterministic, the offset time at a given switch will depend on the processing time of the $\mathrm{BCP}$ in the previous hops. On the other hand, the processing times may not necessarily be deterministic. Consequently, we believe that the offset times at a given switch has a general distribution. Also, we make no assumptions such as low traffic intensity or low blocking probability, and our model is exact.

This article is structured as follows. We start by summarizing Markov fluid queues in Section II. Then, in Section III, we describe feedback Markov fluid queues, namely multi-regime Markov fluid queues and continuous feedback Markov fluid queues, and propose a solution to continuous feedback Markov fluid queues based on the solution of multi-regime Markov fluid queues. Afterwards, we give some examples motivating Markov fluid queue analysis in Section IV. In Section V, we describe the stochastic model for the horizon reservation scheme, which is a continuous feedback Markov fluid queue, and derive its parameters. Then, we give numerical examples related to the horizon reservation scheme in Section VI. Conclusions are given in Section VII.

\section{MARKov Fluid Queues}

A system in which the drift into or out of a buffer of finite or infinite capacity is determined by a Markov process is called a Markov fluid queue (MFQ). The Markov process determining the drift is called the modulating or the background process, which usually is a continuous-time Markov chain (CTMC). An MFQ is a joint process $\{X(t), Z(t)\}$, where $Z(t)$ is the background CTMC, and $X(t)$ is the buffer level. Let $N<$ $\infty$ denote the number of states of $Z(t) . Z(t)$ modulates the MFQ in the following manner. With every state $i, 1 \leq i \leq N$ of $Z(t)$, there is an associated drift, which we will denote with $r_{i}$. When $Z(t)=i, X(t)$ increases (decreases) with rate $r_{i}$ if $r_{i}>0\left(r_{i}<0\right)$. Of course, when $X(t)$ hits 0 , it can be depleted no further. Therefore, for infinite-capacity MFQs, when the state is $Z(t)=i$, we have

$$
\frac{d}{d t} X(t)= \begin{cases}r_{i}, & X(t)>0 \\ \max \left\{r_{i}, 0\right\}, & X(t)=0 .\end{cases}
$$

Similarly, if the buffer is of finite capacity, which we denote by $B$, when the state is $Z(t)=i$, we have

$$
\frac{d}{d t} X(t)= \begin{cases}\min \left\{r_{i}, 0\right\}, & X(t)=B, \\ r_{i}, & B>X(t)>0, \\ \max \left\{r_{i}, 0\right\}, & X(t)=0 .\end{cases}
$$

We define the cdf of the buffer level $X(t)$ as

$$
F(x, t)=\left[\begin{array}{lll}
F_{1}(x, t) & \cdots & F_{N}(x, t)
\end{array}\right],
$$

where

$F_{i}(x, t)=\operatorname{Pr}\{X(t) \leq x, Z(t)=i\}, \quad 1 \leq i \leq N, \quad t \geq 0$,

with $x \in[0, \infty)$ for the infinite buffer and $x \in[0, B]$ for the finite buffer. Assuming that $Z(t)$ is irreducible, the steadystate cdf $F(x)=\lim _{t \rightarrow \infty} F(x, t)$ always exists for the finite buffer case, and it exists under a stability condition for the infinite buffer case. It is well known [14] that the steady-state cdf satisfies

$$
\frac{d}{d x} F(x) R=F(x) Q,
$$

where $Q=\left[q_{i j}\right], 1 \leq i, j \leq N$ is the infinitesimal generator of $Z(t)$, and $R=\operatorname{diag}\left\{r_{1}, \cdots, r_{N}\right\}$ is the diagonal drift matrix.

For the steady-state cdf to exist in the case of infinite buffer, the system should be stable. This means that the mean drift, $\bar{r}=\pi R$ should be negative. Here, $\pi$ is the stationary distribution of $Z(t)$, and satisfies

$$
\begin{aligned}
\pi Q & =\left[\begin{array}{lll}
0 & \cdots & 0
\end{array}\right], \\
\pi \mathbf{1} & =1,
\end{aligned}
$$

where 1 denotes a column vector of ones. When the stability condition is met, there are basically two sets of boundary conditions:

(i) The buffer level $X(t)$ cannot stay at zero for states that have positive drifts, therefore there can be no probability mass at 0 when the $Z(t)$ is in state in which the drift is positive.

(ii) In the solution of the matrix differential equation (1), positive eigenvalues should be suppressed in order to have a stable cdf.

Since the buffer is limited on both ends in the finite buffer case, we do not need a stability condition. There are two sets of boundary conditions:

(i) The buffer level $X(t)$ cannot stay at zero for states that have positive drifts, therefore there can be no probability mass at 0 when the $Z(t)$ is in state in which the drift is positive.

(ii) For the states that have negative drifts, the value of the steady-state cdf at the upper boundary point $B$ should be equal to the corresponding entry of $\pi$.

Spectral method for the solution of MFQ is well known [14]. However, this method is known to exhibit numerical stability problems as shown in [15], in which a novel solution technique is introduced. In this technique, the matrix $Q R^{-1}$, 
with the help of a similarity transform, is partitioned into a block diagonal matrix as

$$
Y^{-1} Q R^{-1} Y=\left[\begin{array}{ccc}
0 & 0 & 0 \\
0 & A_{-} & 0 \\
0 & 0 & A_{+}
\end{array}\right],
$$

where $A_{-}\left(A_{+}\right)$is a square matrix whose eigenvalues lie in the open left (right) half-plane. An efficient algorithm based on Schur decomposition for this partitioning is given in [16]. Denoting different parts of the matrix $Y^{-1}$ with the notation

$$
Y^{-1}=\left[\begin{array}{c}
L_{0} \\
L_{-} \\
L_{+}
\end{array}\right],
$$

the solution to (1) can be written as

$$
F(x)=a_{0} L_{0}+a_{-} e^{A_{-} x} L_{-}+a_{+} e^{-A_{+}(B-x)} L_{+},
$$

where $a_{0}$ is a scalar and $a_{-}$and $a_{+}$are row vectors to be determined from the boundary conditions. As the eigenvalues of $A_{-}$and $-A_{+}$all have negative real parts, numerical stability is assured. We preferred to employ this algorithm throughout this article.

Note that we are assuming $R$ is invertible. If the drift is zero in a state, say $j$, leading to singular $R$, we end up with an algebraic equation for $F_{j}(x)$ in terms of $F_{i}(x), 1 \leq i \leq N, i \neq j$. Therefore, $F_{j}(x)$ can be dropped from the differential equation (1) and computed from the rest of the cdf when the modified differential equation that has an invertiable rate matrix is solved.

\section{Feedback Markov Fluid Queues}

Markov fluid queues whose parameters, i.e. $Q$ and $R$ matrices, depend on the buffer level are called feedback Markov fluid queues. The dependence can be piecewise constant as in the case of multi-regime Markov fluid queues, or continuous as in the case of continuous feedback Markov fluid queues.

\section{A. Multi-regime Markov Fluid Queues}

Consider a MFQ in which the behavior of the system changes when the buffer level exceeds or goes below certain thresholds. These thresholds divide the buffer into "regimes". In each regime, the $Q$ and $R$ matrices take different values. This system can also be viewed as a collection of MFQs confined within the regimes and that interact at the thresholds called regime boundaries. Such systems are named multiregime Markov fluid queues (MRMFQ).

The dynamics of the buffer level in a MRMFQ with $K$ regimes in state $Z(t)=i$ is governed by

$$
\frac{d}{d t} X(t)= \begin{cases}\min \left\{\tilde{r}_{i}^{(k)}, 0\right\}, & X(t)=T^{(K)}=B, \\ r_{i}^{(k)}, & T^{(k)}>X(t)>T^{(k-1)}, \\ \tilde{r}_{i}^{(k)}, & X(t)=T^{(k)}, 0<k<K, \\ \max \left\{\tilde{r}_{i}^{(0)}, 0\right\}, & X(t)=T^{(0)}=0,\end{cases}
$$

where $T^{(k)}, 0 \leq k \leq K$, denote regime boundaries. The dynamics of the infinite buffer case can be written similarly.

Denoting the $Q$ and $R$ matrices belonging to regime $k$ with $Q^{(k)}$ and $R^{(k)}$, it is shown in [16] that the steady-state pdf of the buffer level, $f(x)$, satisfies

$$
\frac{d}{d x} f^{(k)}(x) R^{(k)}=f^{(k)}(x) Q^{(k)}, 1 \leq k \leq K,
$$

along with a set of boundary conditions, which also include the $Q$ and $R$ matrices at each regime boundary $T^{(k)}$ that are denoted by $\tilde{Q}^{(k)}$ and $\tilde{R}^{(k)}$, and the probability mass accummulation vectors for each regime boundary, $c^{(k)}, 0 \leq k \leq K$. We will not list the set of boundary conditions here. Instead, we refer the reader to [16] that also gives the derivation.

To solve a MRMFQ with $K$ regimes, one needs to solve (2), which means solving $K$ MFQs upto constants, and then find out the constants using the boundary conditions.

\section{B. Continuous Feedback Markov Fluid Queues}

In a continuous feedback Markov fluid queue (CFMFQ), the behavior of the background process and the drifts in each state depend on the instantaneous buffer level $x$ in a continuous manner. The steady-state cdf now satisfies [17]

$$
\frac{d}{d x} F(x) R(x)=F(x) Q(x),
$$

which is obviously more difficult an equation to solve than (1). We propose to solve CFMFQs by artificially dividing the buffer into $K$ regimes to obtain a MRMFQ that approximates the CFMFQ at hand. In this study, we opted for a uniform discretization, meaning that for $B<\infty$, we let $T^{(k)}=$ $k B / K, 0 \leq k \leq K$, and for the infinite buffer, after we pick a suitable $T^{(K-1)}$, we let $T^{(k)}=k T^{(K-1)} /(K-1), 0 \leq k \leq$ $K-1$. Then, for each regime boundary, we set

$$
\begin{array}{ll}
\tilde{Q}^{(k)}=Q\left(T^{(k-1)}\right), & 0 \leq k \leq K, \\
\tilde{R}^{(k)}=R\left(T^{(k-1)}\right), & 0 \leq k \leq K,
\end{array}
$$

and for each regime, we set $Q^{(k)}=\tilde{Q}^{(k-1)}$ and $R^{(k)}=$ $\tilde{R}^{(k-1)}, 1 \leq k \leq K$. Then, the method described in [16] is used to solve the system.

The selection of $T^{(K-1)}$ in the infinite buffer case is not straightforward, and we do not attempt to offer a procedure for it here. However, the general principal should be selecting a value that is beyond the point the pdf dies out, assuming that the infinite buffer is stable.

The accuracy of this solution clearly depends on the number of regimes, $K$. Fortunately, the linear equations produced by the boundary conditions lead to a block-banded matrix equation, which can be solved by block LU decomposition [18] in linear time with respect to $K$. This enables us to use large values for $K$, therefore achieving a sufficient level of accuracy. 


\section{Motivating Markov Fluid Queue Analysis}

In this section, we would like to promote the analysis of MFQs by demonstrating its capabilities. We will also introduce the transformation method that will be employed to model the horizon-based reservation in optical burst switching in section $\mathrm{V}$.

Although the mapping from the physical system to the MFQ domain is sometimes obvious like in dam systems, MFQ analysis can be used in a broader set of problems. First, we consider the classical problem of the waiting time in an $\mathrm{M} / \mathrm{M} / 1$ queue. This example is due to [19]. Let the arrival process be Poisson with rate $\lambda$, and the service time be exponentially distributed with mean $1 / \mu$. This system is equivalent to a system that has a service rate of 1 , and the each customer brings an amount workload that is exponentially distributed with mean $1 / \mu$. Then, the waiting time for the $\mathrm{M} / \mathrm{M} / 1$ queue is the remaining workload in the system. Between arrivals, the workload is depleted with a rate of 1 . When an arrival occurs, the workload jumps upwards by an amount brought by the arriving customer. Obviously, we cannot work with such abrupt jumps. Therefore, the jumps are replaced by linear increases of slope 1 . These two behaviors define the two states of the background process of the MFQ. Then, the infinitesimal generator of the background process and the diagonal drift matrix are respectively

$$
Q=\left[\begin{array}{cc}
-\mu & \mu \\
\lambda & -\lambda
\end{array}\right], \quad R=\left[\begin{array}{cc}
1 & 0 \\
0 & -1
\end{array}\right] .
$$

After solving the MFQ with the assumption $\lambda<\mu$, we obtain the pdf of the workload as

$$
f(x)=\left[\begin{array}{ll}
f_{1}(x) & f_{2}(x)
\end{array}\right]=\left[\begin{array}{ll}
1 & 1
\end{array}\right] \lambda \frac{\mu-\lambda}{\mu+\lambda} e^{(\lambda-\mu) x}
$$

along with the probability mass vector at 0

$$
c=\left[\begin{array}{ll}
c_{1} & c_{2}
\end{array}\right]=\left[\begin{array}{ll}
0 & \frac{\mu-\lambda}{\mu+\lambda}
\end{array}\right] .
$$

All that remains at this point to find the workload cdf, $G(x)$, is to remove the spurious state by normalizing the pdf over state 2 to get

$$
G(x)=\frac{c_{2}+\int_{0^{+}}^{x} f_{2}(y) d y}{c_{2}+\int_{0^{+}}^{\infty} f_{2}(y) d y}=1-(\lambda / \mu) e^{(\lambda-\mu) x},
$$

which is the expected result as given in classical texts.

Similar examples could be given such as the derivation of waiting time distribution in an $\mathrm{M} / \mathrm{PH} / 1$ queue based on $\mathrm{MFQ}$ analysis that is given in [20]. Moreover, the MAP/PH/1/1 system can be solved through this method, which would be a special case of the problem attacked in this study with the offset times being 0 for all bursts. Note that the adaptive versions of these problems such as an $\mathrm{M} / \mathrm{M} / 1$ queue in which the arrival rate $\lambda(x)$ depends on the workload through an admission policy, or variations such as customer impatience can also be solved via fluid queue analysis.

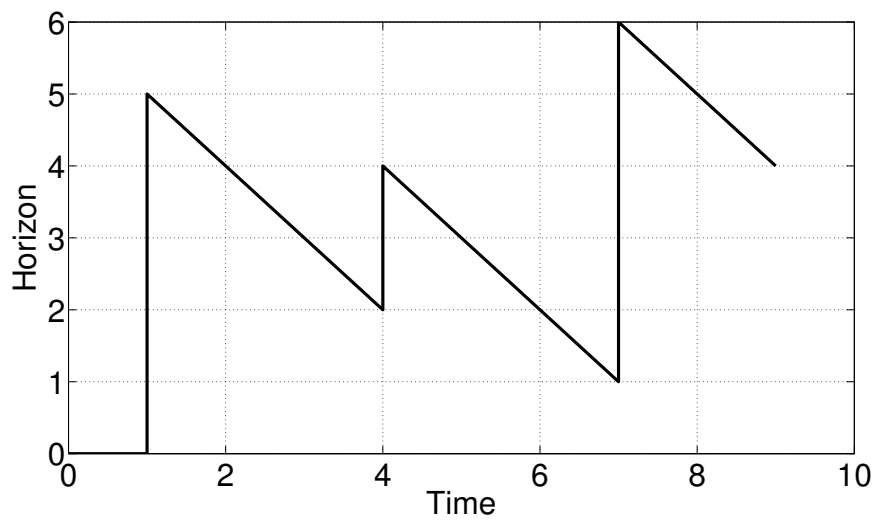

Fig. 1. A sample horizon path with arrivals occuring at $t=1,4,6$ and 7 with offset times 3, 3, 1 and 2, and burst lengths 2, 1, 2 and 4 respectively.

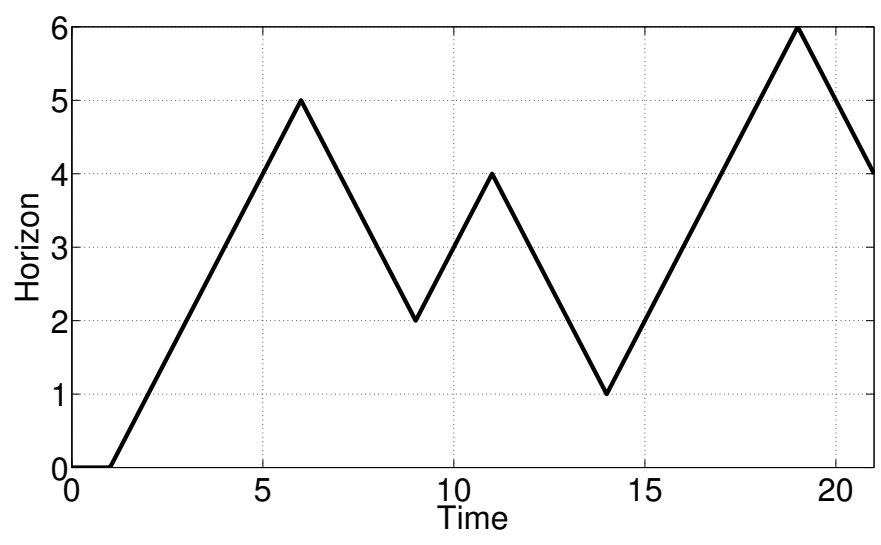

Fig. 2. The transformed horizon path.

\section{Stochastic Model for Horizon-Based OBS}

In horizon-based burst scheduling [4], each optical node maintains a "scheduling horizon" (or just horizon) for each of its interfaces. The horizon is defined as the amount of unfinished work at the designated interface. For each burst, a burst control packet (BCP) is sent out-of-band along the path that the burst is going to traverse in advance of the burst. The BCP contains information about the length of the burst and its offset time, which is the amount of time until the burst will actually arrive at the node.

When a BCP arrives at an optical node, the burst is accepted if the horizon of the node is smaller than the offset time of the burst since the node will not be busy when the burst arrives. In this case, the horizon of the optical node is updated to be equal to the sum of the offset time and the burst length. Otherwise, the burst is said to be blocked.

Consider, for example, four BCPs arriving at an optical node at times $t=1,4,6$ and 7 with offset times 3,3,1 and 2, and burst lengths 2, 1, 2 and 4 respectively. The sample path of the horizon of this optical node is plotted in Fig. 1. The burst corresponding to the third BCP will be blocked since its offset time, 1 , is smaller than the horizon at the time of the arrival of the BCP, which is 2 . The other three bursts will be accepted. 
Proceedings of the 2013 25th International Teletraffic Congress (ITC)

$$
\begin{aligned}
& Q(x)=\left[\begin{array}{ccccc}
I_{\ell_{A} \ell_{B}} \otimes T_{A} & 0 & 0 & 0 & I_{\ell_{A} \ell_{B}} \otimes T_{A}^{0} \\
0 & I_{\ell_{A} \ell_{B}} \otimes T_{B} & 0 & 0 & I_{\ell_{A} \ell_{B}} \otimes T_{B}^{0} \\
H_{A}(x) I_{\ell_{A} \ell_{B}} \otimes \alpha_{A} & 0 & -H_{A}(x) I_{\ell_{A} \ell_{B}} & 0 & 0 \\
0 & H_{B}(x) I_{\ell_{A} \ell_{B}} \otimes \alpha_{B} & 0 & -H_{B}(x) I_{\ell_{A} \ell_{B}} & 0 \\
0 & 0 & I_{\ell_{B}} \otimes D_{1 A}(x) & D_{1 B}(x) \otimes I_{\ell_{A}} & D_{0}(x)
\end{array}\right] \\
& R(x)=R=\left[\begin{array}{ll}
I_{\left(h_{A}+h_{B}+2\right) \ell_{A} \ell_{B}} & \\
& -I_{\ell_{A} \ell_{B}}
\end{array}\right]
\end{aligned}
$$

offset time (denoted by $\tau$ ) expiring in an infinitesimal period between the horizon values $x$ and $x+d x$ given that the offset time "survives" the horizon value $x$ is

$$
\lim _{d x \rightarrow 0} \frac{\operatorname{Pr}\{x<\tau \leq x+d x\}}{\operatorname{Pr}\{\tau>x\}}=H(x) d x .
$$

Fig. 1 transforms into the one given in Fig. 2. This transformed horizon can now be modeled using a CFMFQ. Once the CFMFQ is solved, the states with unity slopes can be censored, i.e. eliminated from the distribution and the distribution of the original system can be obtained by normalizing the pdf over the remaining states.

The background process of the CFMFQ, which is a continuous-time Markov chain, consists of states that correspond to the inter-arrivals, the offset time and the burst length. We assume that the bursts arrive according to a Markovian Arrival Process (MAP) [21] with parameters $D_{0}, D_{1}$. The burst size has a PH-type distribution [22] with parameters $\alpha, T$, and the offset time is generally distributed with $\mathrm{pdf}$ $\theta(x)$ and cdf $\Theta(x)$. Let the MAP have $\ell$ states and the PHtype distribution have $h$ phases (excluding the absorbing state). Then, the CFMFQ is described by the infinitesimal generator

$$
Q(x)=\left[\begin{array}{ccc}
I_{\ell} \otimes T & 0 & I_{\ell} \otimes T^{0} \\
H(x) I_{\ell} \otimes \alpha & -H(x) I_{\ell} & 0 \\
0 & D_{1}(x) & D_{0}(x)
\end{array}\right]
$$

and the diagonal rate matrix

$$
R(x)=R=\left[\begin{array}{ll}
I_{h \ell+\ell} & \\
& -I_{\ell}
\end{array}\right],
$$

where $I_{m}$ denotes the $m \times m$ identity matrix and

$$
\begin{aligned}
T^{0} & =-T \mathbf{1} \\
H(x) & =\frac{\theta(x)}{1-\Theta(x)} \\
D_{1}(x) & =D_{1}(1-\Theta(x)) \\
D_{0}(x) & =D_{0}+D_{1} \Theta(x) .
\end{aligned}
$$

Notice that the $Q(x)$ matrix varies continuously with $x$, resulting in a CFMFQ. This feedback arises due to the system being a blocking system.

The first $h \ell$ states correspond to the PH-type distribution of the burst length, and the next $\ell$ states represent the offset time. The hazard rate given in (8) perfectly models the transition rate out of the offset time states since the probability of the

Note that the hazard rate produces indeterminate values if $\theta(x)$ includes impulses. We do not consider this case in this study, although it is possible to overcome this issue by employing the ideas in [13], which is our ongoing work.

Once the pdf vector of the horizon, denoted by $g(x)$, is found, the burst blocking probability can be computed via

$$
p_{B}=\frac{\int_{0}^{\infty} g(x) D_{1} \mathbf{1} \Theta(x) d x}{\int_{0}^{\infty} g(x) D_{1} \mathbf{1} d x} .
$$

Moreover, the blocking probability conditioned on the offset time can also be computed easily. When the offset time is known to be equal to a known value, say $t_{o}$, the burst will be blocked if the horizon is larger than this value. Hence, the conditional blocking probability is equal to the complementary cumulative distribution function of the horizon:

$$
p_{B \mid \tau=t_{o}}=\int_{t_{o}}^{\infty} g(x) \mathbf{1} d x .
$$

It is also possible to model multiple classes that have different arrival processes, offset time and burst length distributions with this method. Here, we formulate a system with two classes. Formulating systems with more than two classes is not much difficult, although they might lack closed forms. In that case, enumerating the states and building the $Q(x)$ and $R$ matrices algorithmically should be the preferred method. For classes A and B, let the MAP matrix pairs be denoted by $\left(D_{0 A}, D_{1 A}\right)$ and $\left(D_{0 B}, D_{1 B}\right)$ with respective sizes $\ell_{A}$ and $\ell_{B}$, the PH-type parameters by $\left(\alpha_{A}, T_{A}\right)$ and $\left(\alpha_{B}, T_{B}\right)$ with respective number of phases $h_{A}$ and $h_{B}$, and the offset time pdf and cdf's by $\theta_{A}(x), \Theta_{A}(x)$ and $\theta_{B}(x), \Theta_{B}(x)$ respectively. Then, the matrices describing the CFMFQ are given by (4) and (5), where $T_{A}^{0}$ and $T_{B}^{0}$ are defined similar to (7), $H_{A}(x)$ and $H_{B}(x)$ are defined similar to (8), and

$$
\begin{aligned}
D_{1 A}(x) & =D_{1 A}\left(1-\Theta_{A}(x)\right) \\
D_{0 A}(x) & =D_{0 A}+D_{1 A} \Theta_{A}(x) \\
D_{0}(x) & =I_{\ell_{B}} \otimes D_{0 A}(x)+D_{0 B}(x) \otimes I_{\ell_{A}}
\end{aligned}
$$

with similar definitions for $D_{1 B}(x)$ and $D_{0 B}(x)$. 


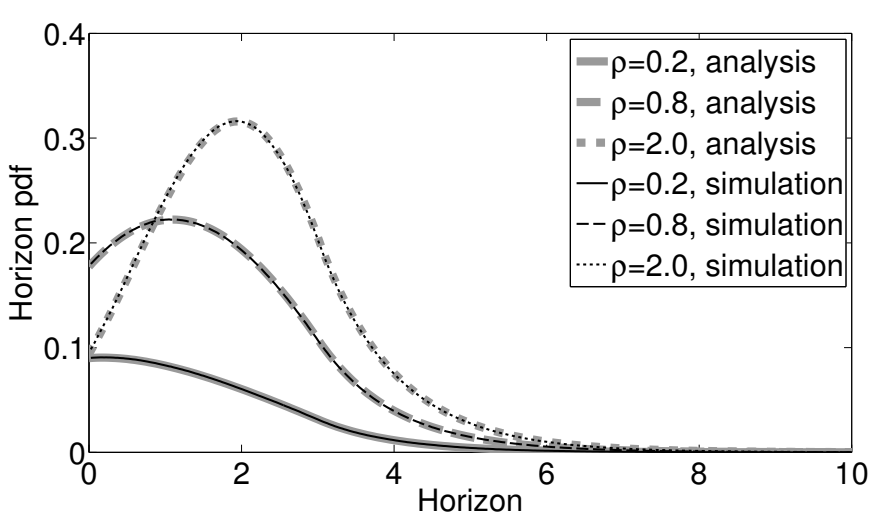

Fig. 3. Horizon pdf plots for 3 different load values.

As a final note, we point out that for a system with $N$ classes, the total number of states of the background process will be $\left(1+N+\sum_{i=1}^{N} h_{i}\right) \prod_{i=1}^{N} \ell_{i}$ with $\prod_{i=1}^{N} \ell_{i}$ of them having -1 drifts.

\section{NUMERICAL EXAMPLES}

First, we start with a single class example in which the arrivals are MAP, the burst lengths are PH-type distributed and the offset times are uniform on $[0,3]$. The matrices defining the MAP are

$$
D_{0}=\left[\begin{array}{cc}
-2 \rho & 0 \\
2 \rho & -2 \rho
\end{array}\right], D_{1}=\left[\begin{array}{cc}
0 & 2 \rho \\
0 & 0
\end{array}\right],
$$

and the parameters of the PH-type distribution are

$$
\alpha=\left[\begin{array}{ll}
0.8 & 0.2
\end{array}\right], T=\left[\begin{array}{cc}
-2 & 1 \\
0 & -1
\end{array}\right] .
$$

The mean of the burst length distribution is 1 , which means that the load is $\rho$. We obtained two sets of results for (i) load values between 0.02 and 0.2 with increments of 0.02 (low loads), and (ii) load values between 0.2 and 2 with increments of 0.2 (moderate to high loads). The pdf plots for the loads $0.2,0.8$ and 2 are given in Fig. 3. The agreement between the analytical and simulated results is obvious, thus validating our method. Within this scenario, we also would like to present the burst blocking probability against load. First, we present in Fig. 4 the burst blocking probability for $\rho=0.02$ computed by our method with various number of regimes. As seen, the blocking probability converges as the number of regimes grow. Thanks to the fact that our method has linear time complexity in the number of regimes, it is possible to use number of regimes as high as $2^{15}$. One can pick a value for the number of regimes in line with their required level of accuracy.

We give the burst blocking probability against load in figures 5 and 6 , for low and moderate to high loads respectively. Figures given in Fig. 5 were obtained with $K=2^{14}$ regimes and $10^{8}$ seconds of simulated time, whereas figures given in Fig. 6 were obtained with $K=2^{10}$ regimes and $10^{6}$ seconds of simulated time. $T^{(K-1)}$ value was selected to be 50 for

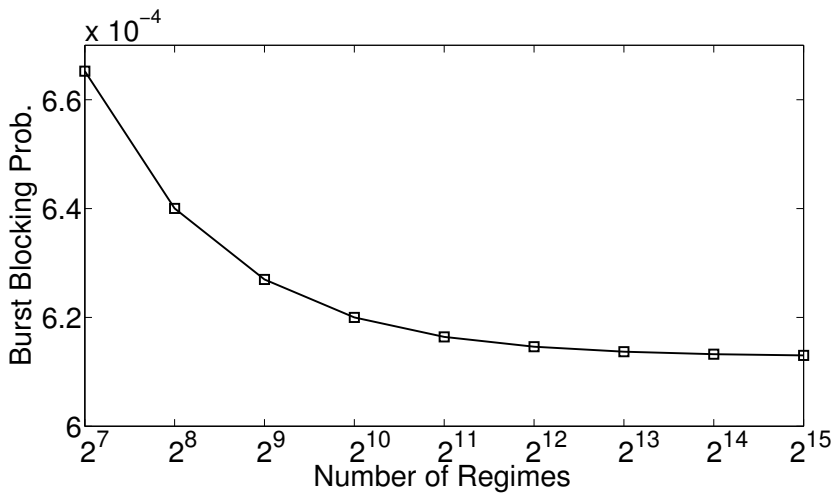

Fig. 4. Burst blocking probability for $\rho=0.02$ found via analysis with different number of regimes.

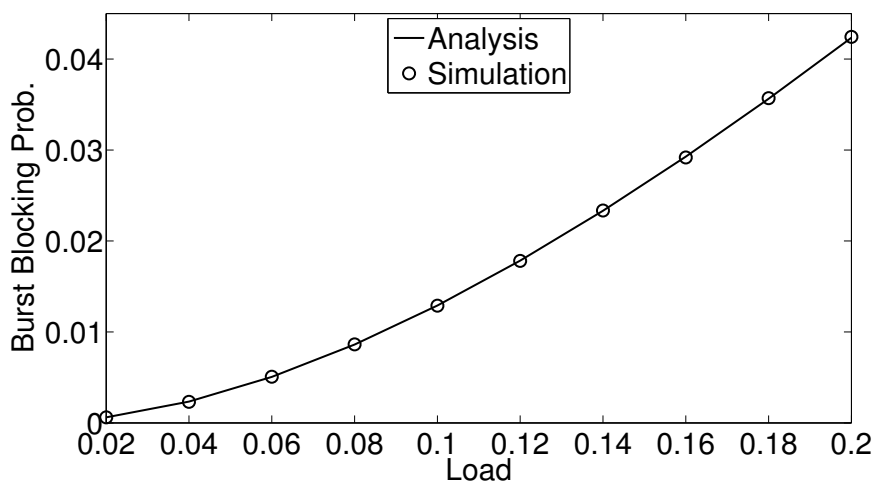

Fig. 5. Burst blocking probabilities for different load values: Low loads. $K=2^{14}$ regimes are used for the analysis and $10^{8}$ seconds were simulated.

all the examples. We also give the burst blocking probability conditioned on the offset time for $\rho=2$ in Fig. 7 .

Next, we investigate the effect of shifting the offset time distribution on the blocking probability. In this example, the arrivals are Poisson with rate 0.1, the burst lengths are exponentially distributed with mean 1 and the offset times are uniformly distributed on intervals of width 5 . We vary the mean of the offset times between 2.5 and 32.5 with increments

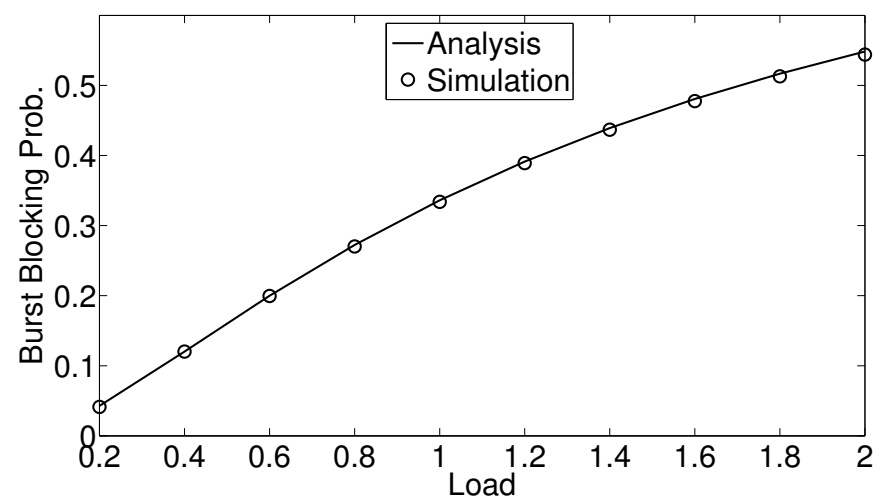

Fig. 6. Burst blocking probabilities for different load values: Moderate to high loads. $K=2^{10}$ regimes are used for the analysis and $10^{6}$ seconds were simulated. 


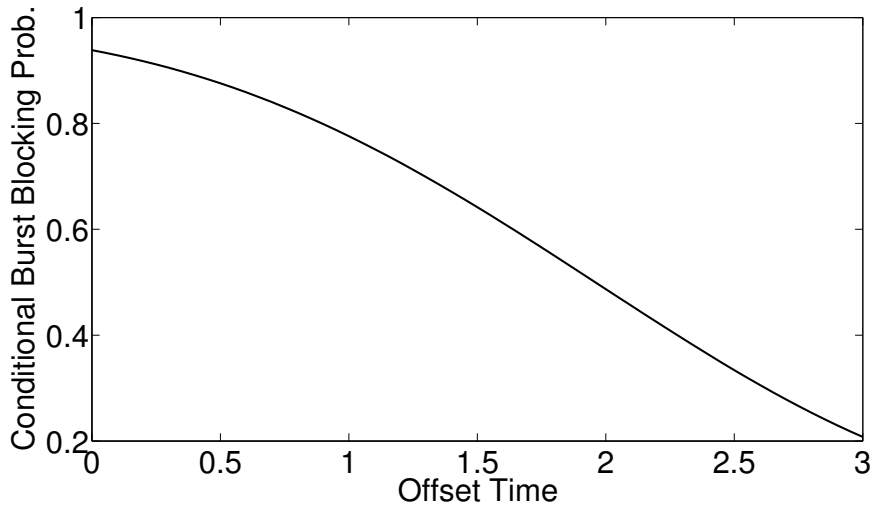

Fig. 7. Burst blocking probability conditioned on the offset time for $\rho=2$.

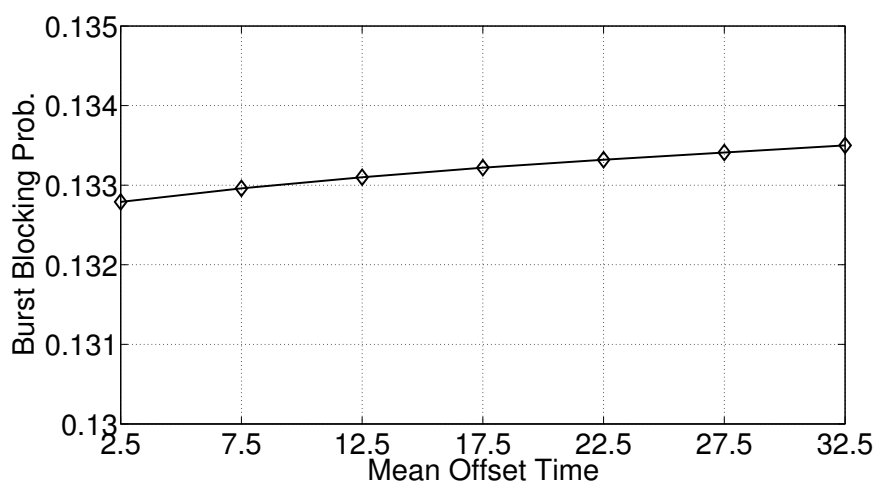

Fig. 8. The effect of shifting the offset time distribution on the blocking probability.

of 5. The blocking probabilities are plotted in Fig. 8. The blocking probabilites barely change, so we conclude that shifting the offset time distribution without changing its shape has only a marginal effect on the blocking probability.

After observing that shifting has a marginal effect, we investigate the effect of the offset time variation. We use the same arrival process and burst length distribution as the previous example. The offset times are uniformly distributed in the interval $[10-u / 2,10+u / 2]$, so the mean offset time is fixed at 10, but the variance increases with the parameter $u$. In Fig. 9, the blocking probability is plotted against $u$, showing that increased variation in offset time hurts the performance in terms of blocking probability, i.e. deterministic offset times are preferable over random ones. This result was also obtained in [9], but through simulation.

Lastly, we give a two-class example. The arrivals of both classes are Poisson and the overall rate is varied between 0.2 and 1 with increments of 0.2 . Three scenarios in which class 1 , the low priority class, brings $25 \%, 50 \%$ and $75 \%$ of the overall traffic are solved. Burst lengths are exponentially distributed with mean 1 for both classes. In this example, we compare two cases. In the first one, the offset times of the two classes are taken to be uniform with very small supports. The offset time of class 1 is uniform in $[0,0.05]$ whereas the offset time of class 2 is uniform in $[5,5.05]$. In the second case, we replace

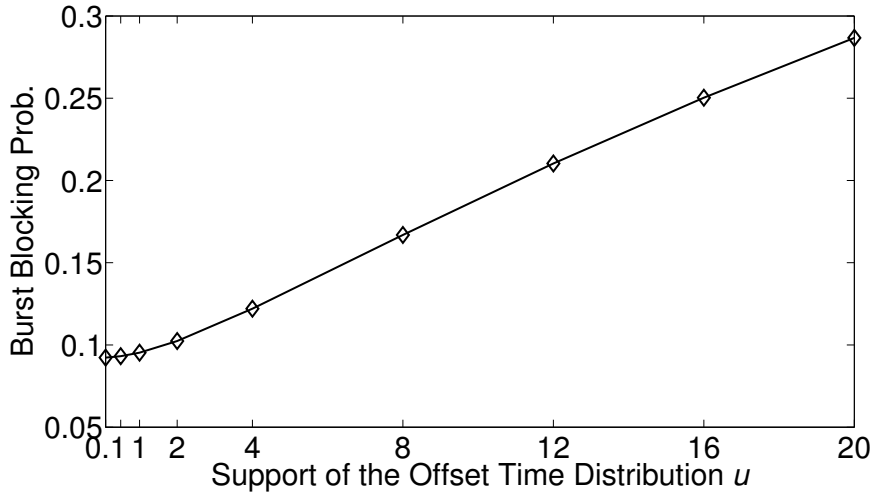

Fig. 9. The effect of shifting the offset time variation.

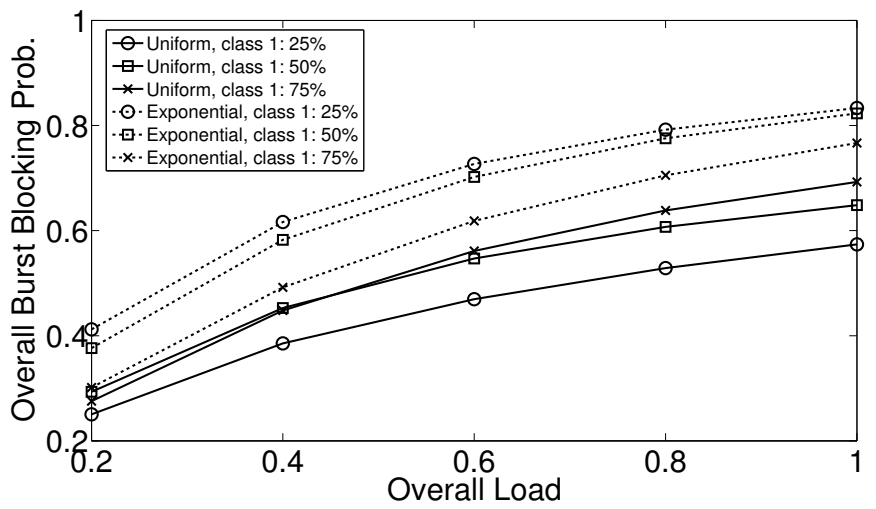

Fig. 10. Overall blocking probability with two classes.

the offset time distribution of class 2 with an exponential distribution with mean 5 . We had showed that keeping the mean constant, offset time distributions with higher variances lead to increased blocking probabilities. Therefore, an increase in the overall blocking probability is to be expected, which is seen in Fig. 10. However, with this example, we intend to investigate the effect of the offset time distribution on the quality of service (QoS) in terms of class separation. For this purpose, we plotted the ratio of the blocking probability of lower priority class to the blocking probability of higher priority class in Fig. 11. We see that not only the exponentially distributed offset time for the higher priority class increases overall blocking probability, it also reduces the separation between the classes. Therefore, we conclude that in addition to achieving lower blocking probabilities, deterministic offset times also lead to better class separation when QoS is concerned.

\section{CONCLUSION}

In this study, we model the horizon reservation scheme on a single-channel OBS system and give an exact solution to the model. We assume no specific circumstances such as low load. In the most general case, the bursts arrive according to a MAP, burst lengths are PH-type distributed and the offset time is generally distributed. 


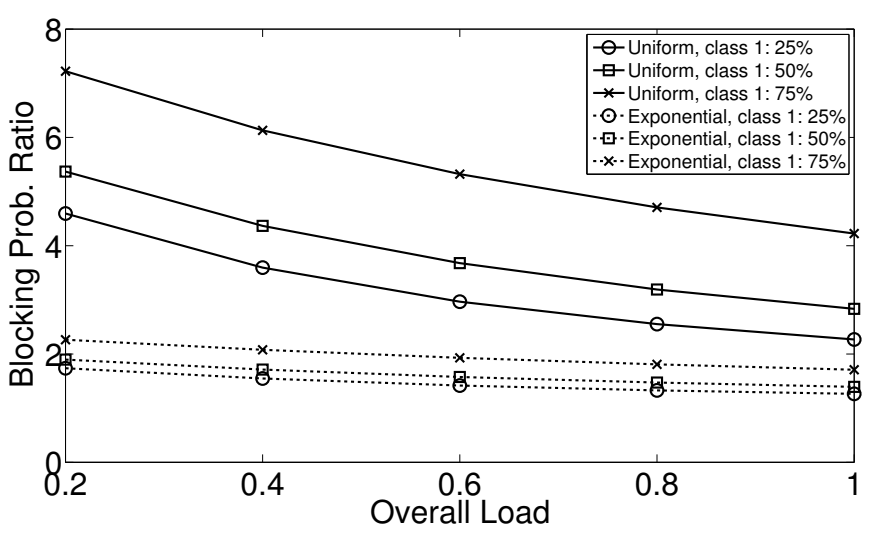

Fig. 11. Ratios of the blocking probabilities of the two classes.

We base our model on the continuous feedback Markov fluid queue (CFMFQ) theory. We describe how the system maps to a CFMFQ by formulating the matrices that describe the CFMFQ. Then, by discretizing the CFMFQ into a MRMFQ, we obtain a much simpler system that can be solved using the method described in [16]. We provide numerical examples validating our model, and also investigate the effect of the offset time distribution on the burst blocking probability. We are currently working on integrating the case of impulsive offset time distributions into our model. Potential extension of the method to multi-channel scenarios is also left for future work.

\section{ACKNOWLEDGMENT}

This work is supported in part by TÜBİTAK (The Scientific and Technological Research Council of Turkey) grant no. 111E106.

\section{REFERENCES}

[1] C. Qiao and M. Yoo, "Optical burst switching (OBS)-A new paradigm for an optical internet," J. High Speed Networks, vol. 8, no. 1, pp. 69-84, 1999.

[2] J. Y. Wei and R. I. McFarland, "Just-In-Time signaling for WDM optical burst switching networks," J. Lightwave Technol., vol. 18, no. 12, p. 2019, December 2000.

[3] M. Yoo and C. Qiao, "Just-Enough-Time (JET): A high speed protocol for bursty traffic in optical networks," in IEEE/LEOS Technologies for a Global Information Infrastructure, August 1997, pp. 26-27.

[4] J. Turner, "Terabit burst switching," J. High Speed Networks, vol. 8, pp. 3-16, 1999.
[5] M. Yoo and C. Qiao, "Supporting multiple classes of services in IP over WDM networks," in Global Telecommunications Conference, 1999. GLOBECOM '99, vol. 1B, 1999, pp. 1023-1027 vol. 1b.

[6] N. Barakat and E. Sargent, "An accurate model for evaluating blocking probabilities in multi-class OBS systems," Communications Letters, IEEE, vol. 8, no. 2, pp. 119-121, February 2004.

[7] D. Morató, M. Izal, J. Aracil, E. Magaña, and J. Miqueleiz, "Blocking time analysis of obs routers with arbitrary burst size distribution," in Global Telecommunications Conference, 2003. GLOBECOM '03. IEEE, vol. 5, December 2003, pp. 2488-2492 vol.5.

[8] H. L. Vu and M. Zukerman, "Blocking probability for priority classes in optical burst switching networks," Communications Letters, IEEE, vol. 6, no. 5, pp. 214-216, May 2002.

[9] K. Dolzer, C. Gauger, J. Späth, and B. Stefan, "Evaluation of reservation mechanisms for optical burst switching," AEU - International Journal of Electronics and Communications, vol. 55, no. 1, pp. 18-26, 2001.

[10] J. Teng and G. N. Rouskas, "A comparison of the JIT, JET, and horizon wavelength reservation schemes on a single OBS node," in In Proc. of the First International Workshop on Optical Burst Switching, 2003, p. 2003.

[11] A. Kaheel, H. Alnuweiri, and F. Gebali, "A new analytical model for computing blocking probability in optical burst switching networks," Selected Areas in Communications, IEEE Journal on, vol. 24, no. 12, pp. 120-128, December 2006.

[12] J. Hernandez, J. Aracil, L. de Pedro, and P. Reviriego, "Analysis of blocking probability of data bursts with continuous-time variable offsets in single-wavelength obs switches," Lightwave Technology, Journal of, vol. 26, no. 12, pp. 1559-1568, June 2008.

[13] H. E. Kankaya and N. Akar, "Exact analysis of offset-based service differentiation in single-channel multi-class OBS," Communications Letters, IEEE, vol. 13, no. 2, pp. 148-150, February 2009.

[14] V. G. Kulkarni, "Fluid models for single buffer systems," in Frontiers in Queuing: Models and Applications in Science and Engineering, J. H. Dshalalow, Ed. CRC Press, 1997, pp. 321-338.

[15] N. Akar and K. Sohraby, "Infinite- and finite-buffer Markov fluid queues: A unified analysis," Journal of Applied Probability, vol. 41, no. 2, pp. 557-569, 2004

[16] H. E. Kankaya and N. Akar, "Solving multi-regime feedback fluid queues," Stochastic Models, vol. 24, no. 3, pp. 425-450, 2008.

[17] W. Scheinhardt, N. van Foreest, and M. R. H. Mandjes, "Continuous feedback fluid queues," Operations Research Letters, vol. 33, no. 6, pp. $551-559,2005$.

[18] G. H. Golub and C. F. van Loan, Matrix Computations. The Johns Hopkins University Press, 1996.

[19] H. E. Kankaya, "Performance evaluation of telecommunication networks based on multi-regime fluid queues," Ph.D. dissertation, Bilkent University, 2009.

[20] T. Dzial, L. Breuer, A. da Silva Soares, G. Latouche, and M.-A. Remiche, "Fluid queues to solve jump processes," Performance Evaluation, vol. 62, pp. 132-146, 2005.

[21] D. M. Lucantoni, K. S. Meier-Hellstern, and M. F. Neuts, "A singleserver queue with server vacations and a class of non-renewal arrival processes," Adv. Appl. Prob., vol. 22, no. 3, pp. 676-705, 1990.

[22] M. F. Neuts, Matrix-Geometric Solutions in Stochastic Models. The Johns Hopkins University Press, 1989. 